\title{
Constitutive model for third harmonic generation in elastic solids
}

\author{
Vamshi Krishna Chillara ${ }^{\mathrm{a}, *}$, Cliff J Lissenden ${ }^{\mathrm{b}}$ \\ ${ }^{a}$ Acoustics and Sensors group, MPA 11, Los Alamos National Laboratory, NM 87545 \\ ${ }^{b}$ Department of Engineering Science and Mechanics, The Pennsylvania State University, \\ 16802 PA
}

\section{Abstract}

In this article, we present a new constitutive model for studying ultrasonic third harmonic generation in elastic solids. The model is hyperelastic in nature with two parameters characterizing the linear elastic material response and two other parameters characterizing the nonlinear response. The limiting response of the model as the nonlinearity parameters tend to zero is shown to be the well-known St Venant-Kirchhoff model. Also, the symmetric response of the model in tension and compression and its role in third harmonic generation is shown. Numerical simulations are carried out to study third harmonic generation in materials characterized by the proposed constitutive model. Predicted third harmonic guided wave generation reveals

\footnotetext{
*Corresponding author

Email address: vchillara@lanl.gov; Ph:814-954-2291 (Vamshi Krishna Chillara )
} 
an increasing third harmonic content with increasing nonlinearity. On the other hand, the second harmonics are independent of the nonlinearity parameters and are generated due to the geometric nonlinearity. The feasibilty of determining the nonlinearity parameters from third harmonic measurements is qualitatively discussed.

\section{Introduction}

Nonlinear ultrasonic methodologies [1] for damage detection are widely being researched with the primary aim of being able to localize and characterize precursors to macro-scale damage. These methodologies employ ultrasound to probe nonlinearity in the material response to characterize the damage. Several techniques like higher harmonic generation [2], Nonlinear elastic wave spectroscopy (NEWS) [3, 4, 5] and Nonlinear resonant ultrasound spectroscopy (NRUS) [6] are developed to detect and characterize damage not easily discernible with conventional ultrasonic methodologies. Of these, ultrasonic higher harmonic generation refers to the generation of higher harmonic frequency components from the primary wave propagating in the material. This generation of higher harmonic frequency components caused by the nonlinear material behavior due to the presence of microscale damage is used to decipher the extent of damage progression in the 
material. Second harmonic generation [7] is widely employed to characterize micro-scale damage in materials, especially metals subject to degradation from fatigue [2], creep [8], radiation damage [9], etc. Many such investigations use bulk-waves that travel in unbounded media to study ultrasonic higher harmonic generation both from theoretical and experimental standpoints. However, there is an increasing interest in the use of nonlinear ultrasonic guided waves [10] and surface waves for early damage detection.

Theoretical investigations concerning the study of nonlinear guided wave propagation in plates [11, 12, 13] and pipes [14, 15] have been carried out by several researchers. Likewise, numerical studies [16, 17] concerning nonlinear guided wave propagation were carried out. However, it should be recognized that the constitutive model used for modeling the nonlinear material behavior in isotropic materials is the same and given by one of the following two equivalent forms of the elastic strain energy function:

\section{Landau-Lifshitz Model}

$$
\mathrm{W}(\mathbf{E})=\frac{1}{2} \lambda(\operatorname{tr}(\mathbf{E}))^{2}+\mu t r\left(\mathbf{E}^{2}\right)+\frac{1}{3} C(\operatorname{tr}(\mathbf{E}))^{3}+B \operatorname{tr}(\mathbf{E}) \operatorname{tr}\left(\mathbf{E}^{2}\right)+\frac{1}{3} \operatorname{Atr}\left(\mathbf{E}^{3}\right)
$$




\section{Murnaghan Model}

$$
\mathrm{W}(\mathbf{E})=\frac{1}{2} \lambda(\operatorname{tr}(\mathbf{E}))^{2}+\mu t r\left(\mathbf{E}^{2}\right)+\frac{1}{3}(l+2 m)(\operatorname{tr}(\mathbf{E}))^{3}-m t r(\mathbf{E})\left((\operatorname{tr}(\mathbf{E}))^{2}-\operatorname{tr}\left(\mathbf{E}^{\mathbf{2}}\right)\right)+n d e t(\mathbf{E})
$$

Here, $\mathbf{E}$ denotes the Lagrangian strain, $\lambda, \mu$ are the Lame's constants while $A, B, C$ are called the third order elastic constants and $l, m, n$ are called the Murnaghan constants. The constants $(A, B, C)$ and $(l, m, n)$ are related by $, l=B+C, m=\frac{1}{2} A+B$ and $n=A[18]$. Both of these models can be regarded as Taylor series expansions (up to third order) of the strain energy function for an isotropic material about the reference state $\mathbf{E}=0$. In the context of nonlinear ultrasonics, the constants $(A, B, C)$ and $(l, m, n)$ can be interpreted as state variables quantifying the extent of nonlinearity in the material behavior [19]. It is easy to see that $A=B=C=0$ corresponds to linear elastic material behavior with geometric nonlinearity. It should be emphasized that the above models possess two main drawbacks with regard to studying nonlinear wave propagation (both bulk and guided waves) which is of interest to nondestructive evaluation and characterization of materials. They are:

1. Due to the third order nature of the Taylor series expansion, the model 
can predict only second harmonic generation in materials for primary stress waves having amplitudes of few MPa. Even in cases with high primary wave amplitudes, second harmonic content dominates third harmonic generation.

2. Also, the three independent higher order constants $(A, B, C)$ or $(l, m, n)$ cannot all be determined from higher harmonic generation measurements as there are only two types of waves namely longitudinal and shear waves propagating in the materials.

In fact some of the recent experimental studies [20, 21] report the generation of third harmonics in materials and their use to characterize material degradation. In this regard, it is worthwhile to consider developing new models especially for modeling third harmonic generation in elastic solids. To that end, this article presents a new constitutive model for studying third harmonic generation in elastic solids. First, some important aspects of the constitutive model development along with the salient features of the constitutive model are discussed. Then we present some numerical studies pertaining to third harmonic generation in materials characterized by the new constitutive model. Finally, conclusions are drawn. 


\section{Constitutive model}

In this section we present the constitutive model and discuss some important aspects of material behavior depicted by the model with regard to nonlinear wave propagation. But first we introduce the notation used in the section. We denote the deformation gradient by $\mathbf{F}$, the displacement gradient by $\mathbf{H}$ and the Lagrangian strain by $\mathbf{E}$. The following relations exist between these kinematic variables, where I denotes the Identity tensor.

$$
\begin{gathered}
\mathbf{F}=\mathbf{I}+\mathbf{H} \\
\mathbf{E}=\frac{1}{2}\left(\mathbf{F}^{\mathbf{T}} \mathbf{F}-\mathbf{I}\right)=\frac{1}{2}\left(\mathbf{H}+\mathbf{H}^{\mathbf{T}}+\mathbf{H}^{\mathbf{T}} \mathbf{H}\right) .
\end{gathered}
$$

The second Piola-Kirchhoff stress is denoted by $\left(\mathbf{T}_{\mathbf{R R}}\right)$. For hyperelastic materials, we denote the strain energy density by $\mathrm{W}$ and we have

$$
\mathbf{T}_{\mathbf{R R}}=\frac{\partial \mathrm{W}}{\partial \mathbf{E}}
$$

The constitutive model we propose for studying third harmonic generation in isotropic materials is given by the strain energy function 


$$
\mathrm{W}(\mathbf{E})=\frac{1}{2 \alpha_{1}}\left(e^{\left(\alpha_{1} \lambda(\operatorname{tr}(\mathbf{E}))^{2}\right)}-1\right)+\frac{1}{\alpha_{2}}\left(e^{\left(\alpha_{2} \mu t r\left(\mathbf{E}^{2}\right)\right)}-1\right)
$$

Here, $\lambda$ and $\mu$ are the Lame's constants and $\alpha_{1}$ and $\alpha_{2}$ are the nonlinearity parameters as will be evident from the discussion to follow. The following observations need to be made regarding the analytical structure of the proposed model $(\operatorname{Eqn}(6))$ :

1. Unlike Eqns(1 \& 2), the strain energy function is written just in terms of two invariants i.e., $\operatorname{tr}(\mathbf{E})$ and $\operatorname{tr}\left(\mathbf{E}^{2}\right)$.

2. The strain energy function is not an explicit Taylor's expansions i.e., it is not in the polynomial form. However, some interesting insights can be obtained by considering the Taylor expansion of the terms in Eqn(6). We illustrate this by considering the Taylor expansion of the terms in $\operatorname{Eqn}(6)$ i.e.,

$$
\frac{1}{2 \alpha_{1}}\left(e^{\left(\alpha_{1} \lambda(\operatorname{tr}(\mathbf{E}))^{2}\right)}-1\right)=\frac{1}{2} \lambda(\operatorname{tr}(\mathbf{E}))^{2}+\frac{1}{2} \frac{\alpha_{1}}{2 !}\left(\lambda(\operatorname{tr}(\mathbf{E}))^{2}\right)^{2}+\frac{1}{2} \frac{\alpha_{1}^{2}}{3 !}\left(\lambda(\operatorname{tr}(\mathbf{E}))^{2}\right)^{3}+\cdots
$$


$\frac{1}{\alpha_{2}}\left(e^{\left(\alpha_{2} \mu t r\left(\mathbf{E}^{2}\right)\right)}-1\right)=\mu \operatorname{tr}\left(\mathbf{E}^{2}\right)+\frac{\alpha_{2}}{2 !}\left(\mu \operatorname{tr}\left(\mathbf{E}^{2}\right)\right)^{2}+\frac{\alpha_{2}^{2}}{3 !}\left(\mu \operatorname{tr}\left(\mathbf{E}^{2}\right)\right)^{3}+\cdots$

- The first term in each of the above equations correspond to the linear elastic material behavior independent of $\alpha_{1}$ and $\alpha_{2}$. Second terms in the expansion are fourth order in $\mathbf{E}$ and are responsible for third harmonic generation. Likewise, the third term is sixth order in $\mathbf{E}$ and is responsible for fifth harmonic generation, and so on for the other terms. So, the proposed model can in fact predict all the odd harmonics generated due to the material nonlinearity. However, our interest is mainly in third harmonic generation.

The corresponding second Piola-Kirchhoff stress for the proposed model obtained from Eqn(5) is given by

$$
\mathbf{T}_{\mathbf{R R}}=\lambda \operatorname{tr}(\mathbf{E})\left(\mathbf{e}^{\left(\alpha_{1} \lambda(\operatorname{tr}(\mathbf{E}))^{2}\right)}\right) \mathbf{I}+2 \mu\left(\mathbf{e}^{\left(\alpha_{2} \mu \operatorname{tr}\left(\mathbf{E}^{2}\right)\right)}\right) \mathbf{E}
$$

Next, we discuss some important features of the proposed model (Eqn (6)). 


\section{Features of the proposed model}

The proposed model has the following important features.

\section{St Venant-Kirchhoff model as the limiting case}

As mentioned earlier, the nonlinearity parameters in the constitutive model $(\operatorname{Eqn}(\sqrt[6]{6})$ represent the extent of micro-scale damage in the context of nonlinear ultrasonics. Hence, the model is constructed under the restriction that the material response is linear elastic (includes geometric nonlinearity) when the nonlinearity parameters $\alpha_{1} \rightarrow 0$ and $\alpha_{2} \rightarrow 0$. In this case, the limiting strain energy function (from $\operatorname{Eqn}(6)$ ) as $\alpha_{1} \rightarrow 0$ and $\alpha_{2} \rightarrow 0$ is given by

$$
\mathrm{W}_{l i n}(\mathbf{E})=\frac{1}{2} \lambda(\operatorname{tr}(\mathbf{E}))^{2}+\mu \operatorname{tr}\left(\mathbf{E}^{2}\right)
$$

This is the well known St Venant-Kirchhoff model in nonlinear hyper elasticity.

\section{Symmetric Tension-Compression response}

It was discussed in [3, 22, 19] that the tension-compression asymmetry in the material response is responsible for even harmonic generation in elastic materials. In addition, a detailed analysis of such asymmetric 
response depicted by Eqns(1 \& 2] was discussed in [22, 19]. Since we are interested in modeling third (odd) harmonic generation in elastic materials, the proposed model $(\operatorname{Eqn}(6))$ is conceived under the restriction that its response is symmetric in tension and compression. For the present discussion this can be stated as $\mathrm{W}(\mathbf{E})=\mathrm{W}(-\mathbf{E})$. Next, we discuss the material response depicted by the constitutive model in $\operatorname{Eqn}(6)$.

We consider the uniaxial stretch deformation given by

$$
x_{1}=s X_{1} ; x_{2}=X_{2} ; x_{3}=X_{3}
$$

where $\left\{x_{i}\right\}_{i=1}^{3}$ denote the coordinates in the current configuration and $\left\{X_{i}\right\}_{i=1}^{3}$ denote the coordinates in the reference configuration. Here $s$ denotes the stretch in the $X_{1}$ direction. The corresponding deformation gradient and the Lagrangian strain are given in matrix representation by 


$$
\mathbf{F}=\left[\begin{array}{ccc}
s & 0 & 0 \\
0 & 1 & 0 \\
0 & 0 & 1
\end{array}\right]
$$

and

$$
\mathbf{E}=\left[\begin{array}{ccc}
\frac{1}{2}\left(s^{2}-1\right) & 0 & 0 \\
0 & 0 & 0 \\
0 & 0 & 0
\end{array}\right]
$$

Figure 1 shows the strain energy function for the uniaxial stretch deformation with $E_{11}$ for different values of $\alpha_{1}=\alpha_{2}=\alpha$ given $\lambda=51$ $\mathrm{GPa}$ and $\mu=26 \mathrm{GPa}$. Also shown is the response for the linear elastic (LE) material that corresponds to $\alpha \rightarrow 0$ (St Venant-Kirchhoff model). As can be seen, the energy is symmetric about $E_{11}=0$ with the energy response approaching the LE case as $\alpha \rightarrow 0$. In addition the stress strain response shown in Figure 2 also shows the symmetry in tension and compression.

\section{Captures both stiffening and softening material responses}

One interesting thing to observe here is that the proposed model is valid even if one or both $\alpha_{1}$ and $\alpha_{2}$ is negative. To illustrate this, we show 
the material response under uniaxial stretch for $\alpha=\alpha_{1}=\alpha_{2}= \pm 4 \mathrm{E}$ $6 \mathrm{~Pa}^{-1}$ in Figure 3. As can be seen, positive $\alpha$ shows a stiffening elastic response and negative $\alpha$ shows a softening response. Also, for negative $\alpha$ the stress-strain response depicts a negative slope (for larger stains not shown in figure) which may be physically unreasonable for elastic response. However, in the context of modeling the nonlinear response from damaged material, it might be interpreted as the onset of macroscale damage beyond which the constitutive model is no longer valid.

\section{Only two nonlinear parameters}

The constitutive model used for studying second harmonic generation (Eqns (1 \& 2) ) has three nonlinear parameters. As emphasized earlier, it should be recognized that all three parameters cannot be determined solely from higher harmonic generation mesurements as there are fundamentally only two waves that propagate in isotropic materials - shear and longitudinal. On the other hand, the proposed constitutive model (Eqn(6) has just two nonlinear parameters, which can be determined from two independent third harmonic generation measurements - one from the longitudinal wave and the other from the shear wave. In fact, 
the parameter $\alpha_{2}$ in the model can be determined from just the shear waves as will be shown later.

\section{Numerical Simulations}

In this section, we present some results from numerical simulations concerning wave propagation in materials governed by the proposed constitutive model. All the simulations are carried out using the solid mechanics module of COMSOL where the constitutive model is incorporated as a user-defined material. First, we present the results obtained for guided wave propagation and then the results for bulk waves are discussed. The linear elastic material properties used for simulations are $\lambda=51 \mathrm{GPa}$ and $\mu=26 \mathrm{GPa}$ and correspond to aluminum with mass density $2700 \mathrm{~kg} / \mathrm{m}^{3}$.

\subsection{Guided waves}

The schematic of the 2D model of a traction-free plate (1 mm thick) used for the simulations is shown in Figure 4. The phase velocity dispersion curves for the plate along with the primary modes to be actuated are shown in Figure 5 .

First, the $\mathrm{S}_{0}$ mode at $0.4 \mathrm{MHz}$ is excited at the left end of the plate by applying through-thickness displacement profiles obtained using the Semi- 
Analytical Finite Element (SAFE) approach 23]. The peak amplitude of the excitation is set to $2 \mathrm{E}-7 \mathrm{~m}$. The third harmonic is the $\mathrm{S}_{0}$ mode at 1.2 $\mathrm{MHz}$ and is not phase-matched to the primary mode. Figure 6 shows the fast Fourier transform (FFT) of time domain signals for the x-component of the displacement on the top surface of the plate at $\mathrm{x}=60 \mathrm{~mm}$ for different nonlinearity parameters $\alpha_{1}=\alpha_{2}=\alpha$. As can be seen, the amplitude of the third harmonic increases with increasing $\alpha$. It is also clear that the second harmonic and the zero-frequency components generated are almost constant and do not change with $\alpha$ indicating that they are associated with the geometric nonlinearity of the finite amplitude excitation. This is also clearly evident in the case where the $\mathrm{S}_{1}$ mode $(3.6 \mathrm{MHz})$ is excited to generate the internally resonant third harmonic $\mathrm{S}_{3}$ mode $(10.8 \mathrm{MHz})$ 24] shown in Figure 7. The amplitude of the excitation is set to $2 \mathrm{E}-8 \mathrm{~m}$. Similar to the case of the $\mathrm{S}_{0}$ mode, the second harmonic and the zero-frequency component do not depend on $\alpha$ whereas the third harmonic increases with increasing $\alpha$. Figure 8 shows the comparison of the FFT's for third harmonic generation from the $\mathrm{S}_{1}$ mode at $3.6 \mathrm{MHz}$ for $\alpha= \pm 16 \mathrm{E}-6 \mathrm{~Pa}^{-1}$. The harmonic content is the same irrespective of the sign of $\alpha$. While the material response is very different for different signs of $\alpha$ as indicated in Figure 3 , it does not affect 
the harmonic content as the amplitude of the stress waves here is quite small $(<10 \mathrm{MPa})$.

\subsection{Bulk shear wave propagation}

Simulations for bulk shear wave propagation in the $\mathrm{x}$-direction are carried out using a 2D plane-strain model shown in Figure 9. Excitation is specified as displacement boundary condition on $50 \mathrm{~mm}$ of the left end highlighted in red. Absorbing boundary conditions are applied on either side as indicated. The amplitude of the displacement boundary condition is set to $2 \mathrm{E}-7 \mathrm{~m}$ for a $0.5 \mathrm{MHz}$ excitation. Simulations are run for different combinations of the nonlinear parameters $\alpha_{1}$ and $\alpha_{2}$ as shown in Figure 10. The third harmonic content does not depend on the parameter $\alpha_{1}$, but it does depend on $\alpha_{2}$. This indicates that the parameter $\alpha_{2}$ can be independently determined from third harmonic generation of shear waves. Also note that there is no second harmonic or zero frequency component. This is because the FFT shown is for the y-component of the displacement and the second harmonic corresponds to a longitudinal wave that only has the $\mathrm{x}$-component of the displacement as it is polarized perpendicular to the primary shear wave. While it is not possible for the bulk shear wave to generate a second harmonic shear wave,

it does generate a second harmonic longitudinal wave [25, 26]. 


\section{Conclusions}

In this article, a new constitutive model was developed to model third harmonic generation in hyperelastic solids. The important features of the constitutive model are:

1. it reduces to the well-known St Venant-Kirchhoff model as the limiting case when the nonlinearity parameters tend to zero;

2. it shows symmetric response in tension and compression;

3. it captures both stiffening and softening material responses;

4. it has only two nonlinearity parameters that can be determined from two third harmonic measurements using bulk waves.

Each of the above features was discussed and numerical simulations were carried out to demonstrate these in the context of nonlinear wave propagation i.e., third harmonic generation. It was found that even though the (material) nonlinearity in the constitutive model is responsible for third harmonic generation, second harmonics are also generated due to the presence of geometric nonlinearity. However, this second harmonic generation is independent of the nonlinearity parameters. Also, the third harmonic content 
seems to be insensitive to the stiffening (positive $\alpha$ ) and softening responses (negative $\alpha$ ) of the materials governed by the proposed model due to the small amplitude of the stress waves in the materials. Simulations for third harmonic generation from bulk shear wave propagation were carried out for different values of the nonlinearity parameters. It was shown that the third harmonic generation from bulk shear waves can be used to determine the pa-

rameter $\alpha_{2}$ in the model $(\operatorname{Eqn}(\sqrt[6]{6})$. Hence, it should be possible to determine both the nonlinearity parameters using the third harmonic generation from both shear and longitudinal waves in the material. We conclude the article by commenting that the constitutive model introduced in this article bears resemblance to the widely used Fung's model ([27]) in biomechanics in terms of the exponential terms used. Hence it appears that the proposed model has a wider range of applicability in other areas of mechanics that need to be explored.

\section{Acknowledgements}

This material is based upon work supported by the National Science Foundation under Award number 1300562. Vamshi Chillara also acknowledges the support from the Penn State College of Engineering in the form of 
Distinguished Teaching Fellowship. 


\section{References}

[1] Y. Zheng, R. G. Maev, I. Y. Solodov, Review/sythèse nonlinear acoustic applications for material characterization: A review, Canadian Journal of Physics 77 (12) (2000) 927-967.

[2] J. H. Cantrell, W. T. Yost, Nonlinear ultrasonic characterization of fatigue microstructures, International Journal of fatigue 23 (2001) 487490.

[3] K.-A. Van Den Abeele, P. A. Johnson, A. Sutin, Nonlinear elastic wave spectroscopy (news) techniques to discern material damage, part i: nonlinear wave modulation spectroscopy (nwms), Research in nondestructive evaluation 12 (1) (2000) 17-30.

[4] K.-A. Van Den Abeele, J. Carmeliet, J. A. Ten Cate, P. A. Johnson, Nonlinear elastic wave spectroscopy (news) techniques to discern material damage, part ii: Single-mode nonlinear resonance acoustic spectroscopy, Journal of Research in Nondestructive Evaluation 12 (1) (2000) 31-42.

[5] K. E. Van Den Abeele, A. Sutin, J. Carmeliet, P. A. Johnson, Microdamage diagnostics using nonlinear elastic wave spectroscopy (news), Ndt \& E International 34 (4) (2001) 239-248. 
[6] M. Muller, A. Sutin, R. Guyer, M. Talmant, P. Laugier, P. A. Johnson, Nonlinear resonant ultrasound spectroscopy (nrus) applied to damage assessment in bone, The Journal of the Acoustical Society of America 118 (6) (2005) 3946-3952.

[7] K. Matlack, J.-Y. Kim, L. Jacobs, J. Qu, Review of second harmonic generation measurement techniques for material state determination in metals, Journal of Nondestructive Evaluation 34 (1) (2014) 1-23.

[8] J. S. Valluri, K. Balasubramaniam, R. V. Prakash, Creep damage characterization using non-linear ultrasonic techniques, Acta Materialia 58 (6) (2010) 2079-2090.

[9] K. Matlack, J. Wall, J.-Y. Kim, J. Qu, L. Jacobs, H.-W. Viehrig, Evaluation of radiation damage using nonlinear ultrasound, Journal of Applied Physics 111 (5) (2012) 054911.

[10] V. K. Chillara, C. J. Lissenden, Review of nonlinear ultrasonic guided wave nondestructive evaluation: theory, numerics, and experiments, Optical Engineering 55 (1) (2016) 011002-011002.

[11] M. Deng, Cumulative second-harmonic generation of lamb-mode prop- 
agation in a solid plate, Journal of applied physics 85 (6) (1999) 30513058 .

[12] W. De Lima, M. Hamilton, Finite-amplitude waves in isotropic elastic plates, Journal of sound and vibration 265 (4) (2003) 819-839.

[13] V. K. Chillara, C. J. Lissenden, Interaction of guided wave modes in isotropic weakly nonlinear elastic plates: Higher harmonic generation, Journal of Applied Physics 111 (12) (2012) 124909.

[14] V. K. Chillara, C. J. Lissenden, Analysis of second harmonic guided waves in pipes using a large-radius asymptotic approximation for axissymmetric longitudinal modes, Ultrasonics 53 (4) (2013) 862-869.

[15] Y. Liu, C. J. Lissenden, J. L. Rose, Higher order interaction of elastic waves in weakly nonlinear hollow circular cylinders. i. analytical foundation, Journal of Applied Physics 115 (21) (2014) 214901.

[16] V. K. Chillara, C. J. Lissenden, Nonlinear guided waves in plates: A numerical perspective, Ultrasonics 54 (6) (2014) 1553-1558.

[17] M. J. Leamy, T. B. Autrusson, W. J. Staszewski, T. Uhl, P. Packo, Local computational strategies for predicting wave propagation in non- 
linear media, in: SPIE Smart Structures and Materials+ Nondestructive Evaluation and Health Monitoring, International Society for Optics and Photonics, 2014, pp. 90641J-90641J.

[18] M. Destrade, R. W. Ogden, On the third-and fourth-order constants of incompressible isotropic elasticity, The Journal of the Acoustical Society of America 128 (6) (2010) 3334-3343.

[19] V. K. Chillara, C. J. Lissenden, On some aspects of material behavior relating microstructure and ultrasonic higher harmonic generation, International Journal of Engineering Science 94 (2015) 59-70.

[20] C. J. Lissenden, Y. Liu, V. K. Chillara, G. Choi, X. Yao, Nonlinear guided waves for continuous material microstructure state awareness, in: ASME 2014 International Mechanical Engineering Congress and Exposition, American Society of Mechanical Engineers, 2014, pp. V013T16A033-V013T16A033.

[21] C. Lissenden, Y. Liu, G. Choi, X. Yao, Effect of localized microstructure evolution on higher harmonic generation of guided waves, Journal of Nondestructive Evaluation 33 (2) (2014) 178-186.

[22] V. K. Chillara, C. J. Lissenden, Towards a micro-mechanics based un- 
derstanding of ultrasonic higher harmonic generation, in: SPIE Smart Structures and Materials+ Nondestructive Evaluation and Health Monitoring, International Society for Optics and Photonics, 2015, pp. 94380R-94380R.

[23] T. Hayashi, W.-J. Song, J. L. Rose, Guided wave dispersion curves for a bar with an arbitrary cross-section, a rod and rail example, Ultrasonics 41 (3) (2003) 175-183.

[24] Y. Liu, V. K. Chillara, C. J. Lissenden, J. L. Rose, Third harmonic shear horizontal and rayleigh lamb waves in weakly nonlinear plates, Journal of Applied Physics 114 (11) (2013) 114908.

[25] Z. Goldberg, Interaction of plane longitudinal and transverse elastic waves, Sov. Phys. Acoust 6 (1961) 306-310.

[26] Y. Liu, V. K. Chillara, C. J. Lissenden, On selection of primary modes for generation of strong internally resonant second harmonics in plate, Journal of Sound and Vibration 332 (19) (2013) 4517-4528.

[27] Y. Fung, K. Fronek, P. Patitucci, Pseudoelasticity of arteries and the choice of its mathematical expression, American Journal of PhysiologyHeart and Circulatory Physiology 237 (5) (1979) H620-H631. 


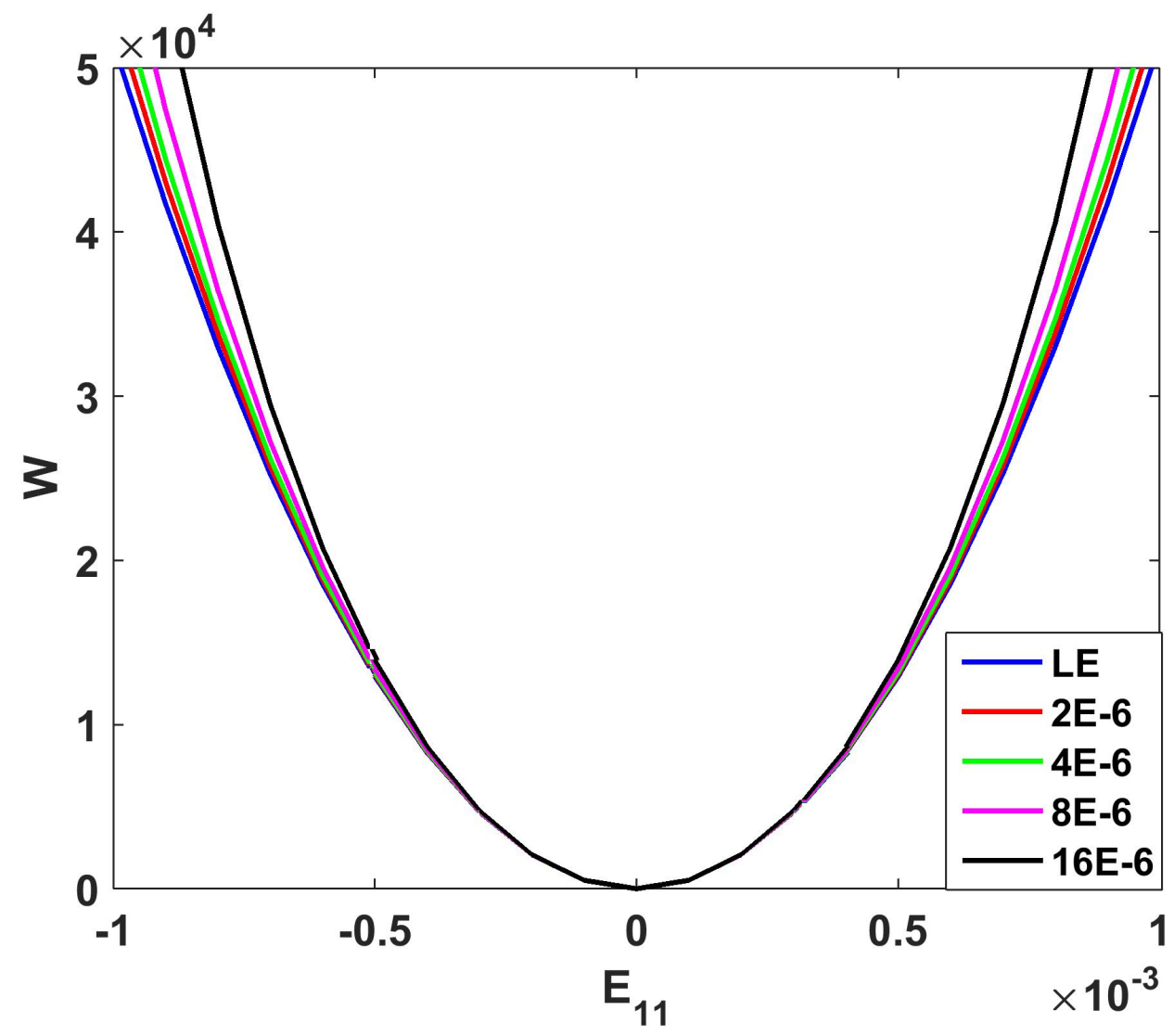

Figure 1: Strain energy density (in Pa) under a uniaxial stretch for different $\alpha_{1}=\alpha_{2}=\alpha$ (in $\mathrm{Pa}^{-1}$ ). 


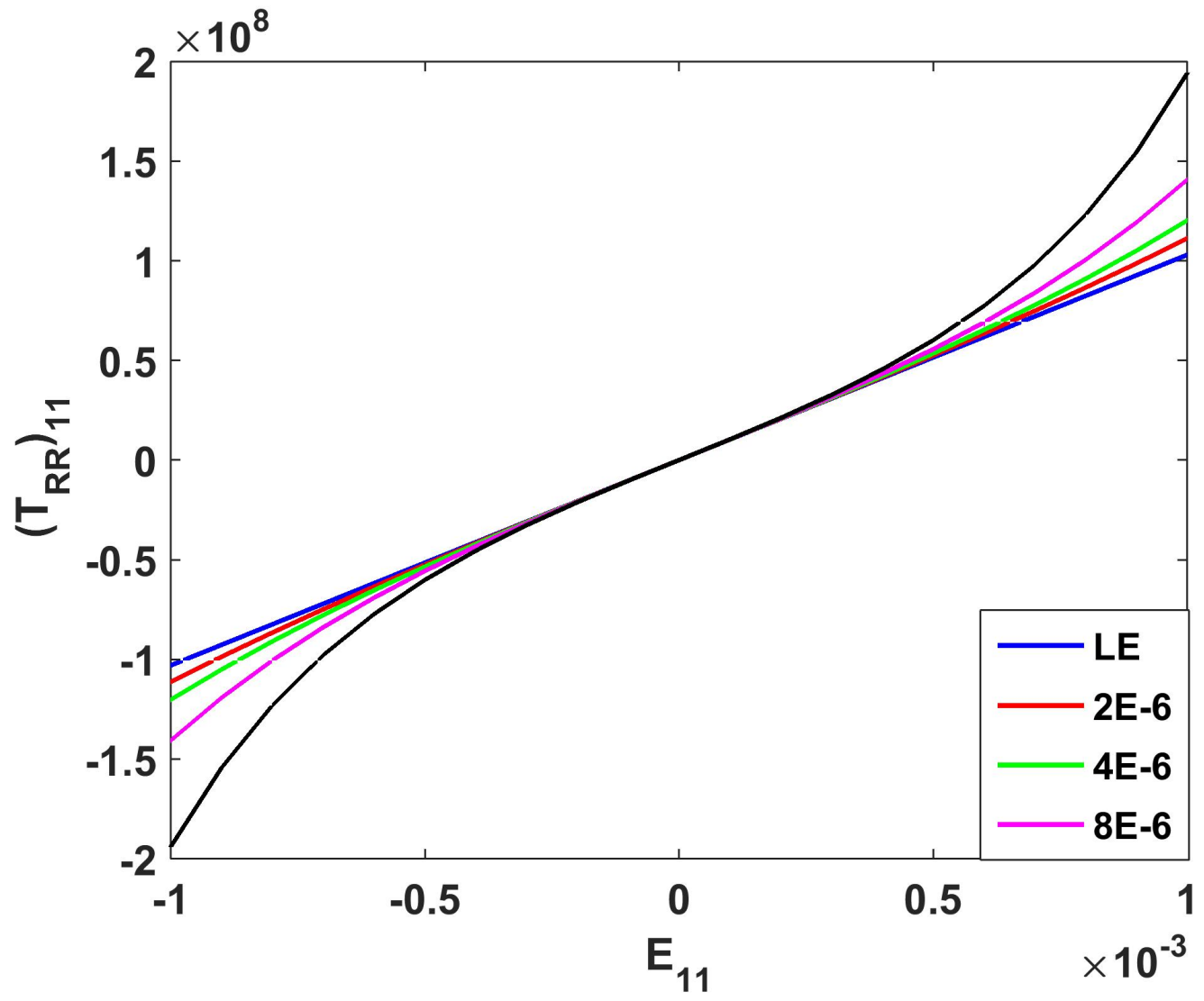

Figure 2: $\left(\mathbf{T}_{\mathbf{R R}}\right)_{\mathbf{1 1}}$ (in Pa) under a uniaxial stretch for different $\alpha_{1}=\alpha_{2}=\alpha\left(\right.$ in $\left.\mathrm{Pa}^{-1}\right)$. 


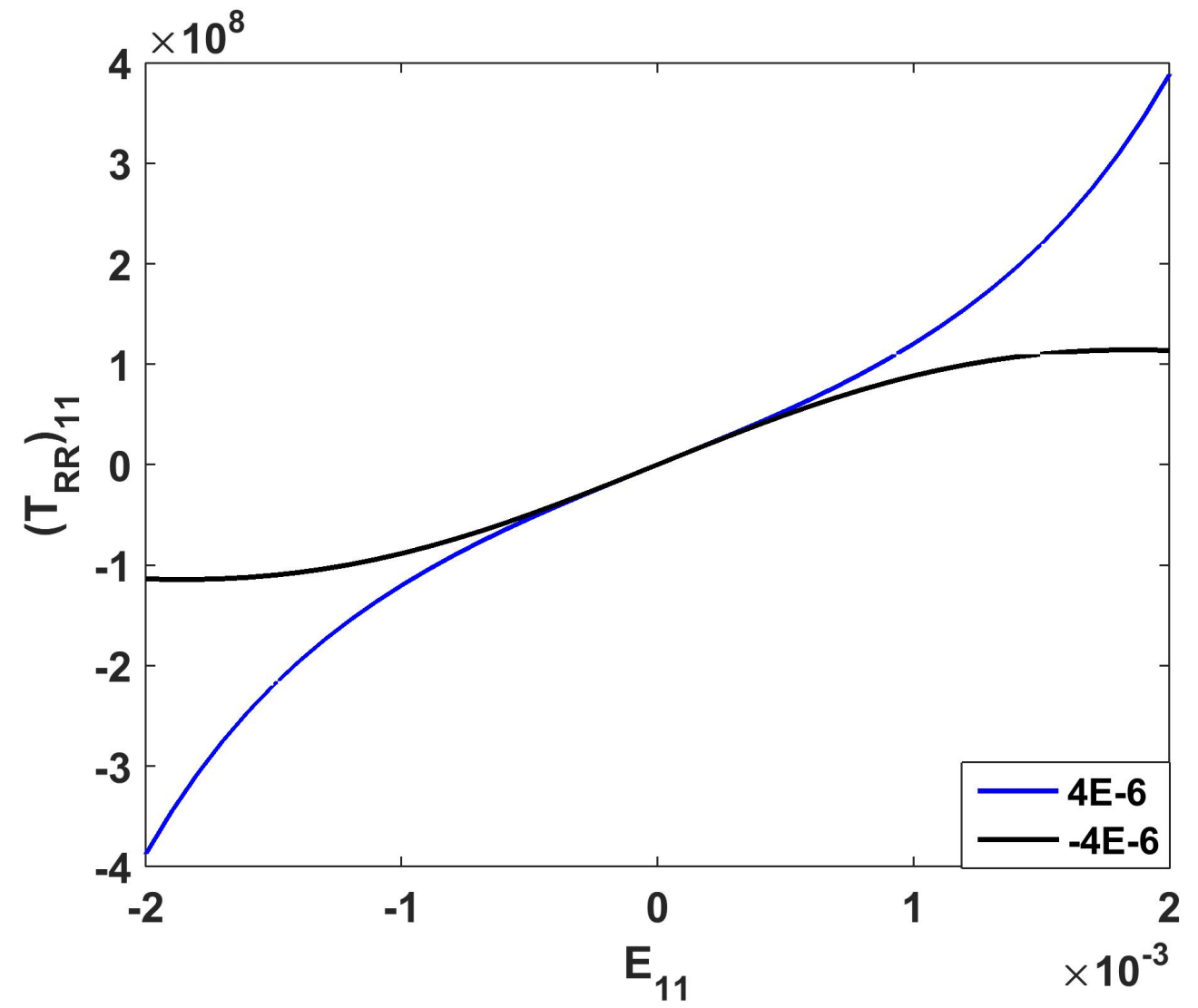

Figure 3: $\left(\mathbf{T}_{\mathbf{R R}}\right)_{\mathbf{1 1}}$ (in Pa) under a uniaxial stretch for different $\alpha= \pm 4 \mathrm{E}-6 \mathrm{~Pa}$.

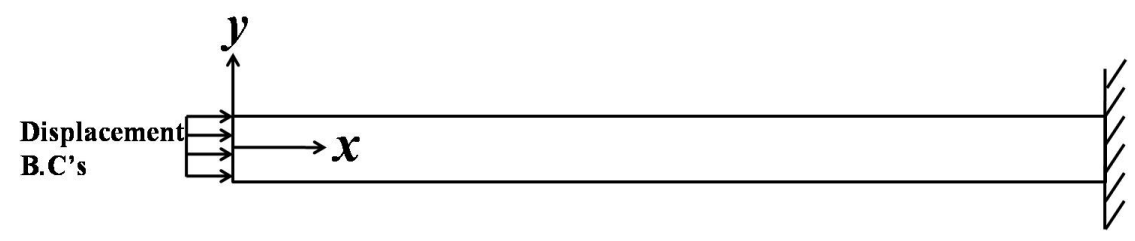

Figure 4: Schematic of the model used for guided wave propagation. 


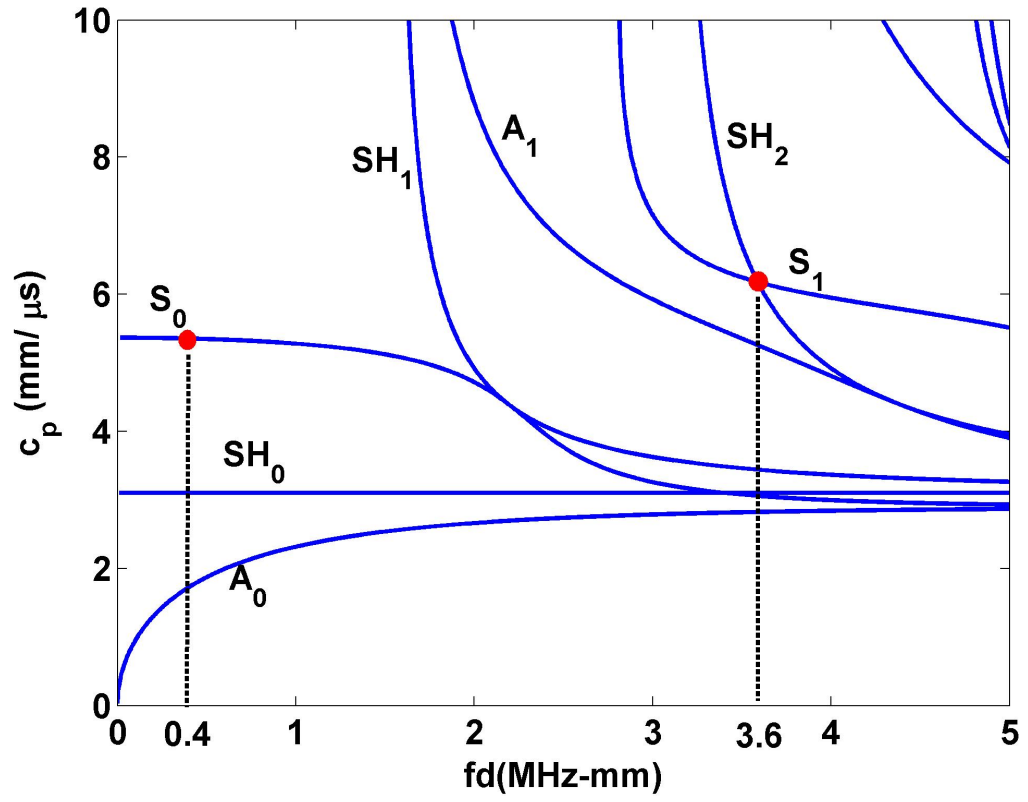

Figure 5: Dispersion curves for the aluminum plate along with the primary modes used for numerical simulations highlighted in red. 


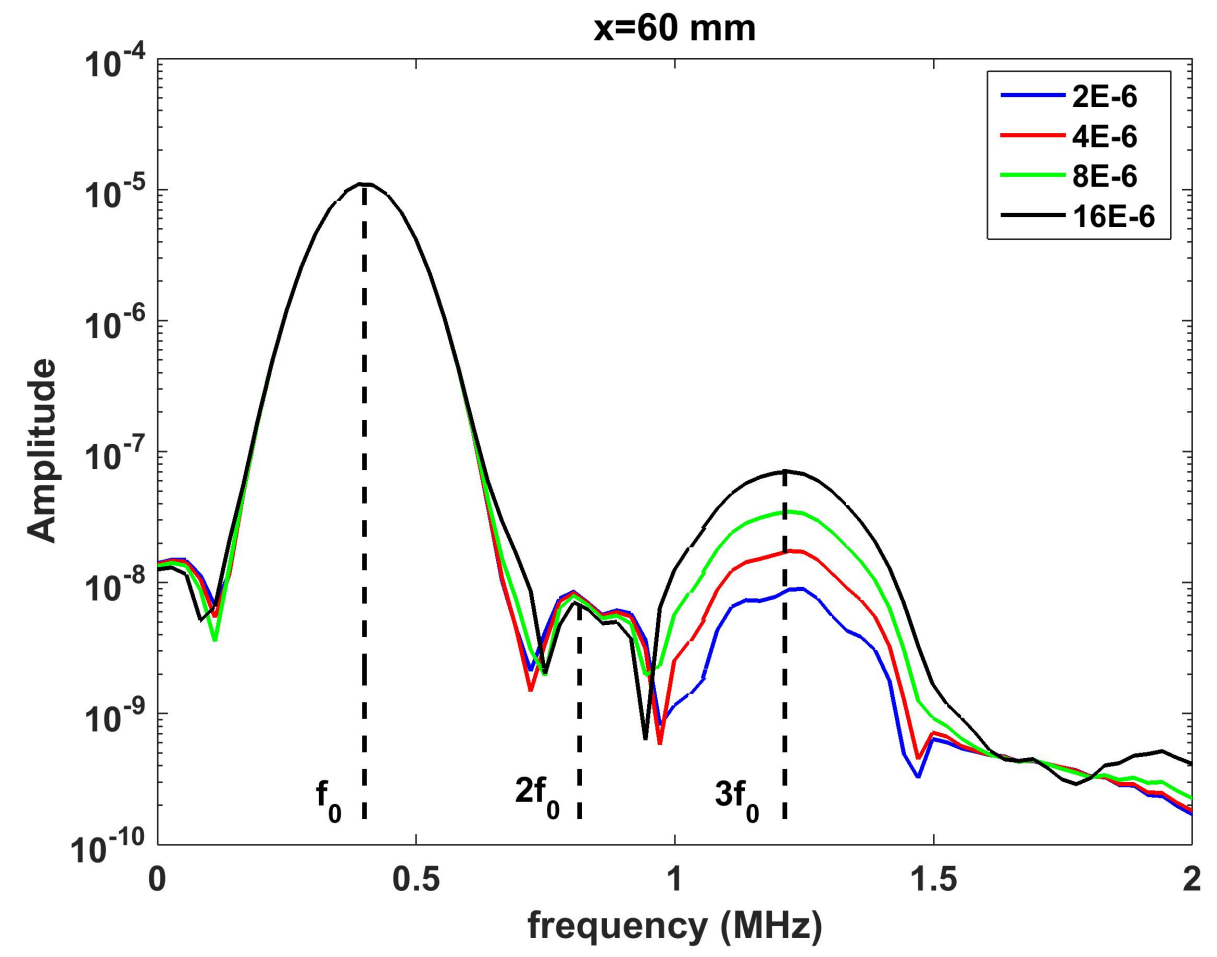

Figure 6: FFT of time-domain signal received at $x=60 \mathrm{~mm}$ for the $\mathrm{S}_{0}$ mode $(0.4 \mathrm{MHz})$ and different $\alpha_{1}=\alpha_{2}=\alpha\left(\right.$ in $\left.\mathrm{Pa}^{-1}\right)$. 


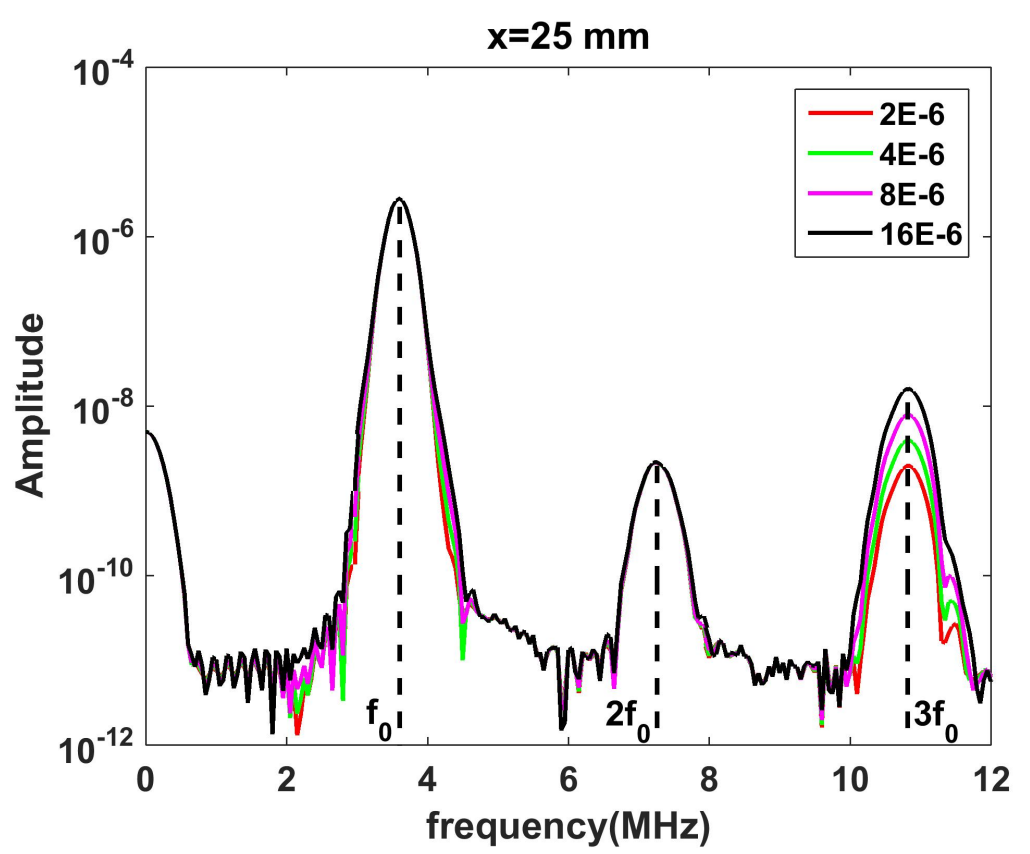

Figure 7: FFT of time-domain signal received at $x=25 \mathrm{~mm}$ for the $\mathrm{S}_{1}$ mode $(3.6 \mathrm{MHz})$ and different $\alpha_{1}=\alpha_{2}=\alpha\left(\right.$ in $\left.\mathrm{Pa}^{-1}\right)$. 


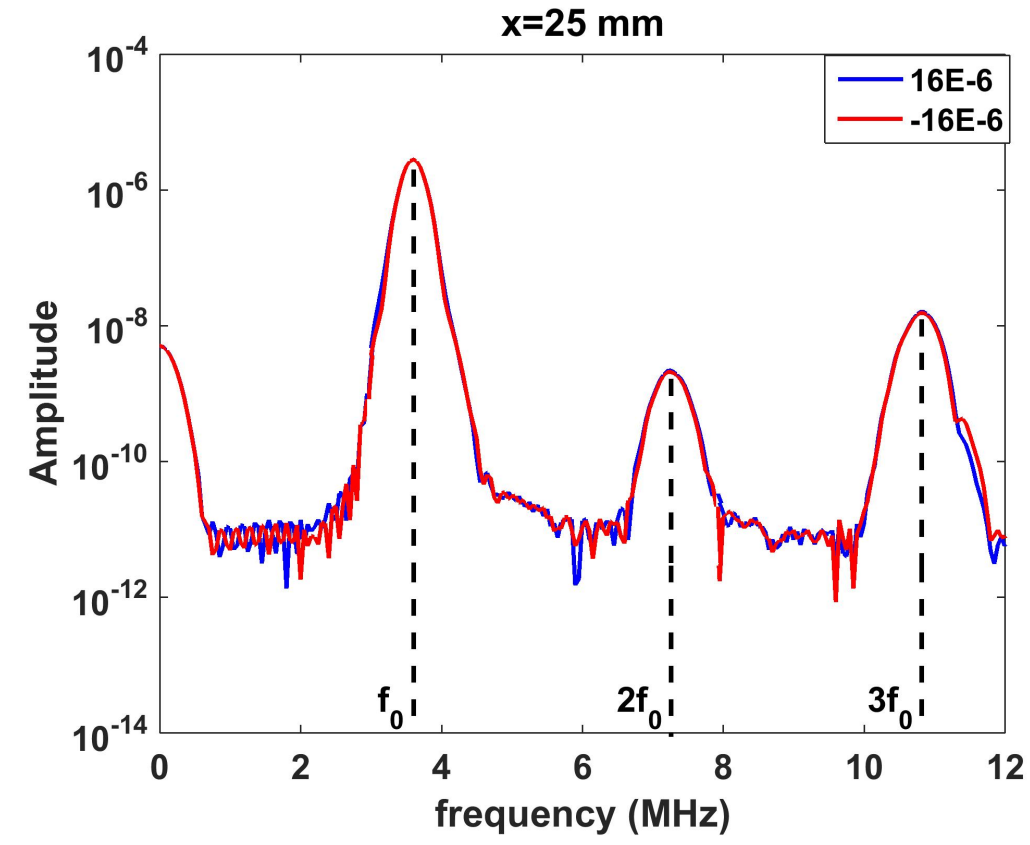

Figure 8: FFT of time-domain signal received at $x=25 \mathrm{~mm}$ for the $\mathrm{S}_{1}$ mode $(3.6 \mathrm{MHz})$ and two different $\alpha= \pm 16 \mathrm{E}-6 \mathrm{~Pa}^{-1}$.

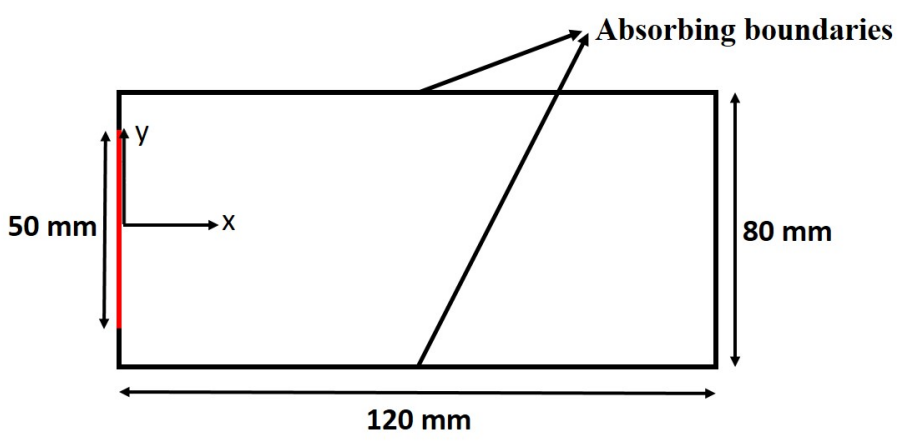

Figure 9: Schematic of the model used for studying bulk shear wave propagation. 


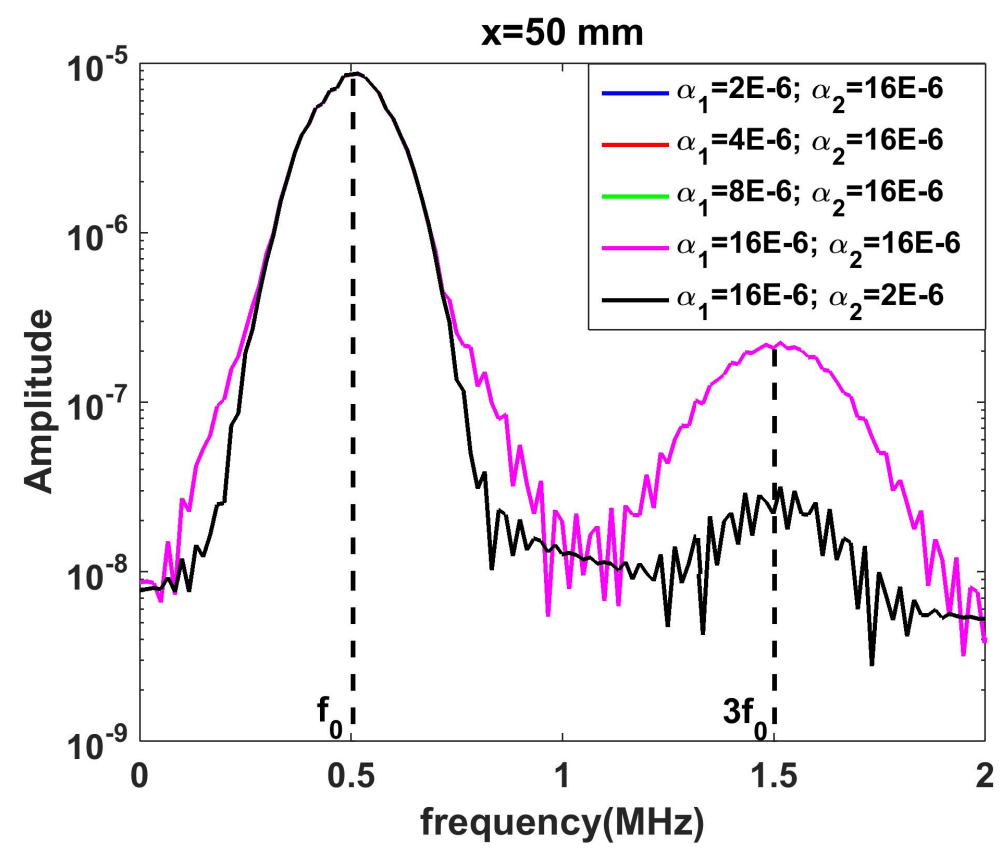

Figure 10: Bulk shear wave $(0.5 \mathrm{MHz}):$ FFT of time-domain signal at $x=25 \mathrm{~mm}, \mathrm{y}=0$ $\mathrm{mm}$ for different $\alpha_{1}$ (in $\mathrm{Pa}^{-1}$ ) and $\alpha_{2}$ (in $\mathrm{Pa}^{-1}$ ) (All plots with the same $\alpha_{2}$ are coincident). 